\title{
Erratum zu: Mit dem Propheten Muhammad in den Islamischen Religionsunterricht - Theologische Impulse für eine erfahrungsbezogene Prophetologie
}

Ruggero Vimercati Sanseverino

\section{Erratum zu:}

Kapitel „Mit dem Propheten Muhammad in den Islamischen Religionsunterricht - Theologische Impulse für eine erfahrungsbezogene Prophetologie" In: R. Vimercati Sanseverino (Hrsg.), Islamische Bildungsarbeit in der Schule, https://doi.org/10.1007/9783-658-26720-9_5

„Durch einen Fehler während der Produktion wurden in diesem Kapitel die Wörter „Prophetologie“ und ,prophetologisch“ an mehreren Stellen durch „Proktologie“ und ,proktologisch“ ersetzt. Dies wurde nun korrigiert.“

Die korrigierte Version des Kapitels ist verfügbar unter https://doi.org/10.1007/978-3-658-26720-9_5

R. V. Sanseverino $(\bowtie)$

Universität Tübingen, Tübingen, Deutschland

E-Mail: ruggero.vimercati-sanseverino@uni-tuebingen.de

(C) Springer Fachmedien Wiesbaden GmbH, ein Teil von Springer Nature 2021 